\section{Is the Nature Index at odds with DORA?}

We find Nature Research's critical attitude towards journal impact factors, embodied in its signing of the San Francisco Declaration on Research Assessment (DORA; Nature 544, $394 ; 2017)$, to be inconsistent with the aims of its Nature Index.

The Nature Index provides statistics on the publication output of institutions and countries. These statistics are collated from "high-quality research" published in an independently selected set of 68 "high-quality science journals" (see www.natureindex. $\mathrm{com} / \mathrm{faq}$ ). The data are presented as metrics that can be used to assess "research excellence and institutional performance".

This seems to us to be in violation of DORA's principles, which state that research should be assessed "on its own merits rather than on the basis of the journal in which the research is published" (www.ascb.org/dora).

Even though we disagree with some of the ideas underlying DORA (see L. Waltman and V. A. Traag, preprint at https:// arxiv.org/abs/1703.02334;2017), we believe that Nature Research should practice what it preaches and abandon the Nature Index.

Ludo Waltman, Vincent Traag Leiden University, the Netherlands. waltmanlr@cwts.leidenuniv.nl

On behalf of the Nature Index, David Swinbanks replies The Nature Index provides an indicator of an institution's contributions to high-quality research, on the basis of papers from a suite of journals that exhibit high editorial standards and have been independently chosen by a committee of active researchers - without regard to impact factor (see go.nature. com/2qo53pj). The Nature Index enables the tracking of institutional research output and collaboration at the city, state and country levels.
It is therefore not in violation of DORA, which champions appropriate ways of appraising individual researchers and opposes the abuse of the journal impact factor as a metric for research assessment.

\section{Don't derail cod's comeback in Canada}

We urge Canada's government not to act on proposals to imminently ramp up the fishery for northern cod (Gadus morhua) along Newfoundland and Labrador's east coast. Although the stock has made a remarkable comeback since its collapse in the early 1990 s - in parallel with other factors such as a greater abundance of cod's key prey, capelin (Mallotus villosus) - numbers are still well below historical norms.

A decision to increase cod fishing would fly in the face of scientists' recommendations under the Canadian government's decision-making framework for fisheries, which uses a precautionary approach. Their advice includes encouraging ecosystem-based management that recognizes the importance of food webs, and keeping fish removals as low as possible until the stock surpasses the minimum abundance needed for optimal production.

After last year's stock assessment, modified management practices allowed the catch to double - even though no rebuilding plan was in place to provide target reference points, timelines or harvest-control rules. There was no stock assessment this spring, despite the advice of the Canadian parliamentary fisheries committee for annual assessments. Meanwhile, the available data indicate that the cod's comeback may have stalled (see go.nature.com/2r9stje).

Signs of a pending decline in cod productivity include lower inshore catch rates and smaller increases in surveyed biomass. The rise in capelin might also have slowed. We therefore strongly advise against stepping up fishing for northern cod at this time. Doing so risks derailing long-term stock recovery and a rebuilt fishery. Sherrylynn Rowe, George A. Rose Memorial University of Newfoundland, St. John's, Canada. sherrylynn.rowe@mi.mun.ca

\section{Species disconnected from DNA sequences}

DNA sequences are the bedrock of molecular taxonomy and phylogenetics. Alarmingly, we find that $20 \%$ of the reptilian sequences in GenBank's DNA database cannot be mapped to actual species or subspecies.

Using the Reptile Database (see go.nature.com/2pgkdbw), we investigated how many taxa in the taxonomic database of the US National Center for Biology Information (NCBI) could be mapped to accepted species. We found that just $60 \%$ of the 10,510 species in the Reptile Database have their taxon identified in the NCBI database. Moreover, 1,704 reptile names in NCBI (19.5\% of all reptile NCBI names) did not match up with their currently accepted species' name in the Reptile Database.

Sloppy practice by authors and journals contributes to these discrepancies. We found that 1,037 species names in published papers were either not rigorously spelled out (for example, the lizard Eremias grammica has been designated as Eremias sp. TMT-2004a) or had not been updated in either GenBank or the NCBI after publication of their DNA sequence.

The problem is likely to get worse as metagenomic and DNA-barcoding studies proliferate. For the sake of the international biodiversity community, we urge authors of taxonomic papers to lodge and update their DNA sequences and the associated taxonomic information in the relevant databases.

Peter Uetz Virginia Commonwealth University, Richmond, USA.

Akhil Garg McGill University, Montreal, Canada. uetz@vcu.edu

\section{Predatory journals: outwit with a safe list}

Urologists have taken a stance against predatory publishers by compiling a 'green list' of reputable journals within the speciality (see https:// urologygreenlist.wordpress.com). Urology researchers are invited to recommend journals that should be included on or removed from the list, and to send supporting evidence that will be judged by an international advisory panel. I strongly encourage all research disciplines to create their own green lists.

Such lists seem less likely to result in litigation and are simple to maintain, unlike the task of blacklisting an ever-increasing number of predatory journals (see also V. J. Giglio and O. J. Luiz Nature 544, 416; 2017, and W. Strielkowski Nature 544, 416; 2017). The absence of a journal from the green list does not necessarily indicate that it is a predatory publication, but I would advise authors to confirm a journal's credentials before submitting a manuscript.

The urologists' green list currently contains 52 bona fide journals (including two owned by Springer Nature, the publishers of Nature). We still need to define the criteria for inclusion more clearly, but factors such as association with a professional society, indexing, quality of the editorial board and history are a good start.

Henry Woo University of Sydney, Australia.

henry.woo@sydney.edu.au Competing interests declared: see go.nature.com/2rtnpjg. 